\title{
Central Retinal Vein Occlusion in a 46-Year-Old Man with COVID-19: Case Report and Review of the Literature
}

\author{
Charles G. Miller Benjamin J. Kim \\ Department of Ophthalmology, Scheie Eye Institute, University of Pennsylvania, \\ Philadelphia, PA, USA
}

\section{Keywords}

Central retinal vein occlusion · Coronavirus · COVID19 · Hypercoagulability · Severe acute respiratory syndrome coronavirus 2

\begin{abstract}
A 46-year-old man with a history of well-controlled hypertension presented with a central retinal vein occlusion (CRVO) in his right eye, which was complicated by cystoid macular edema. When the patient noted new visual symptoms, he was also experiencing muscle aches and easy fatiguability. A standard hypercoagulability panel failed to identify an etiology for his CRVO. However, the patient underwent COVID-19 antibody testing, which returned positive. The patient received a series of aflibercept injections for his macular edema, and his vision improved. Further study is warranted to determine if there is any association between mild infection with COVID-19 and the development of CRVO.
\end{abstract}

\section{Introduction}

The novel severe acute respiratory syndrome coronavirus 2 (SARS-CoV-2 or COVID-19) is known to cause thromboembolic complications [1]. Many such reports have focused on critically ill patients, where the prevalence of venous thromboembolic events has been found to be as high as $27 \%$ [2]. Microangiopathic retinal changes have been observed [3], but whether these are due to prolonged hypoxemia as opposed to a more direct viral etiology is not clear [4]. Furthermore, corresponding hyperreflective foci on optical coherence tomography (OCT) have been suggested as an associated finding but may be unrelated to COVID-19 $[3,5,6]$. Several cases of retinal vein occlusion (RVO) have been attributed to COVID-19 (Table 1), although no large-scale studies have been performed to establish causality [7-12].

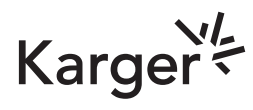


Miller and Kim: Central Retinal Vein Occlusion in a 46-Year-Old Man with COVID-19

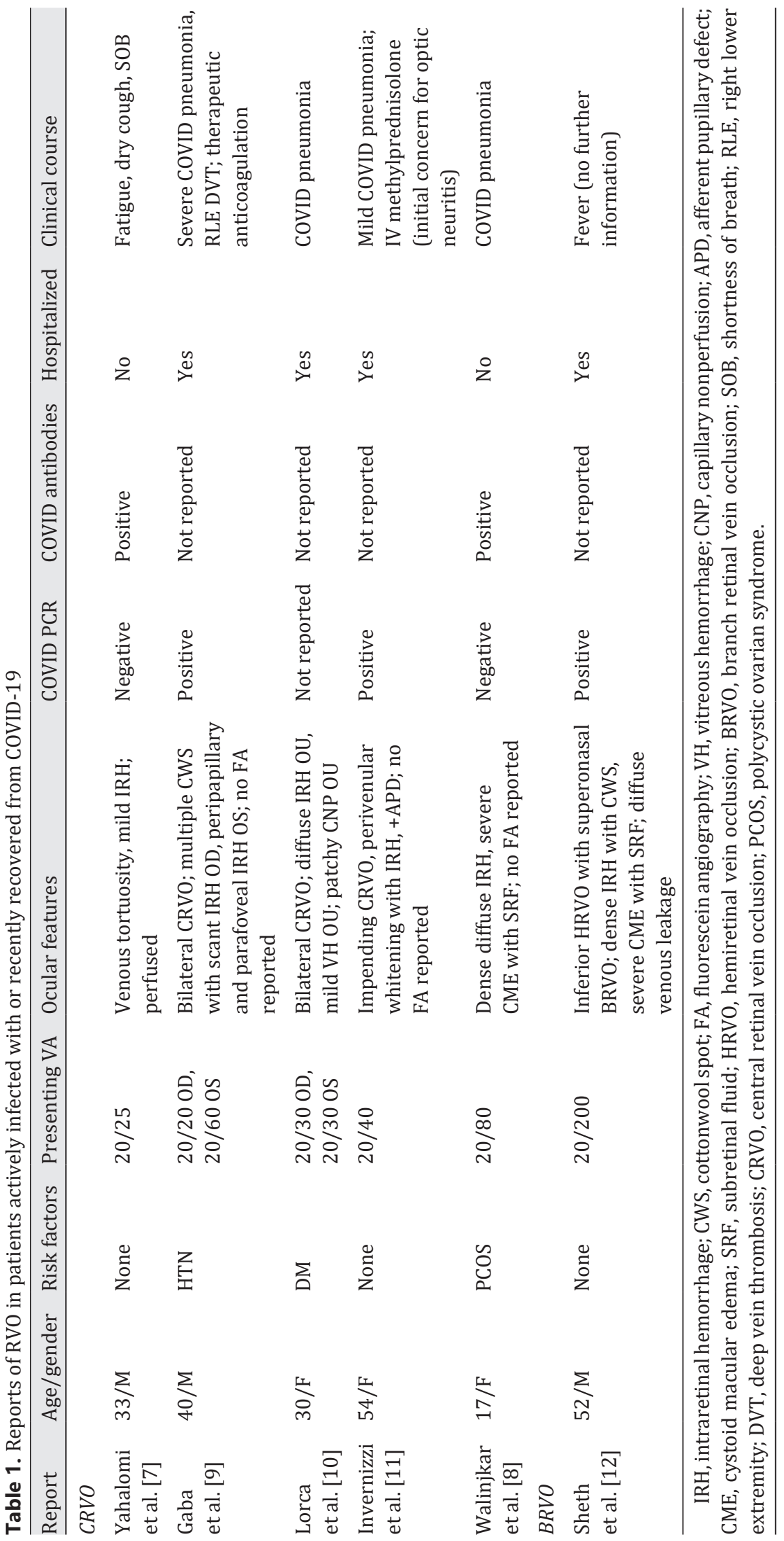


Notably, retina specialists frequently consider pursuing a panel of testing for hypercoagulability risk factors when a young patient presents with an acute RVO. While the diagnostic yield of routine testing has been questioned, many practitioners send a panel of blood tests for individuals younger than 56 years newly diagnosed with vein occlusion [13]. This practice grew out of the observation that patients in this younger age-group may be more likely to harbor an identifiable cause for their thrombophilia [14, 15].

\section{Case Report}

A 46-year-old man with a history of well-controlled hypertension and hyperlipidemia presented for a televisit with his comprehensive ophthalmologist complaining of a "black dot" that suddenly appeared in the central vision of his right eye. He denied any associated visual symptoms, including flashes of light, floaters, or a dark curtain over his vision. The patient did endorse constitutional symptoms over the past 1 month, including muscle aches and easy fatiguability. Based primarily on his visual symptoms, he was advised to present for an in-person evaluation. Uncorrected visual acuity was found to be 20/20 in both eyes, and intraocular pressures were $14 \mathrm{~mm} \mathrm{Hg}$ (right eye) and $12 \mathrm{~mm} \mathrm{Hg}$ (left eye). There were no abnormalities on examination of the anterior segment. His dilated funduscopic examination of the right eye (Fig. 1a) was notable for clear vitreous, mild edema of the optic nerve head, extensive intraretinal hemorrhages in the macula, marked vessel tortuosity, and intraretinal hemorrhages along with cotton wool spots in all 4 quadrants. The left eye had a normal posterior segment. OCT of the macula in the right eye showed intraretinal fluid (Fig. 1b). Other OCT-derived biomarkers of ischemia, including disorganization of the retinal inner layers or paracentral acute middle maculopathy, were absent. The patient underwent further imaging by fluorescein angiogram, which demonstrated diffuse venous leakage and peripheral capillary nonperfusion (Fig. 1c). Based on the totality of findings, a diagnosis of central RVO (CRVO) was made. The patient was then referred to the retina service for further management.

At his first follow-up visit, 11 days later, the patient's visual acuity in the right eye had declined to 20/80+. Based on the patient's relatively young age, he wanted to proceed with further diagnostic testing to determine an etiology for his CRVO, despite his known cardiovascular risk factors of hypertension and hyperlipidemia. A broad hypercoagulability panel was sent (Table 2) including complete blood count, plasma homocysteine level, antiphospholipid antibody panel (anticardiolipin IgG and IgM, anti- $\beta 2$ glycoprotein 1 IgG and IgM, tissue thromboplastin inhibition test, and dilute Russell viper venom time), and proteins $C$ and $S$ levels. The results of all of these tests were normal. In light of the patient's declining visual acuity and with persistent cystoid macular edema again demonstrated on an OCT of the macula, treatment was initiated with intravitreal aflibercept ( $2 \mathrm{mg} / 0.05 \mathrm{~mL})$.

Three weeks after this visit, having never uncovered a reason for his constitutional symptoms, of his own volition, the patient sought out a COVID-19 serum antibody test (SARSCoV-2 antibodies, nucleocapsid; LabCorp) through an urgent care center. This test is an immunoassay with the ability to detect IgG, IgM, and IgA antibodies against COVID-19. The manufacturer's supporting materials and an independent study both indicate sensitivity and specificity $>99 \%$ for COVID-19 in patients $>28$ days after exposure [16, 17]. He never underwent viral PCR testing and never manifested with symptoms specific for COVID-19 including fever, cough, or anosmia. The COVID-19 antibody test returned positive.

Six weeks after his first aflibercept injection, the patient presented for follow-up examination, and his vision in the right eye had returned to 20/20 with resolution of macular edema on OCT (Fig. 1d). Review of the patient's OCT images did not reveal any unusual findings that

\section{Karger'}




\section{Case Reports in Ophthalmology}

\begin{tabular}{l|l}
\hline Case Rep Ophthalmol 2021;12:646-652 \\
\hline DOI: 10.1159/000517417 & $\begin{array}{l}\text { ○ 2021 The Author(s). Published by S. Karger AG, Basel } \\
\text { www.karger.com/cop }\end{array}$ \\
\hline
\end{tabular}

Miller and Kim: Central Retinal Vein Occlusion in a 46-Year-Old Man with COVID-19
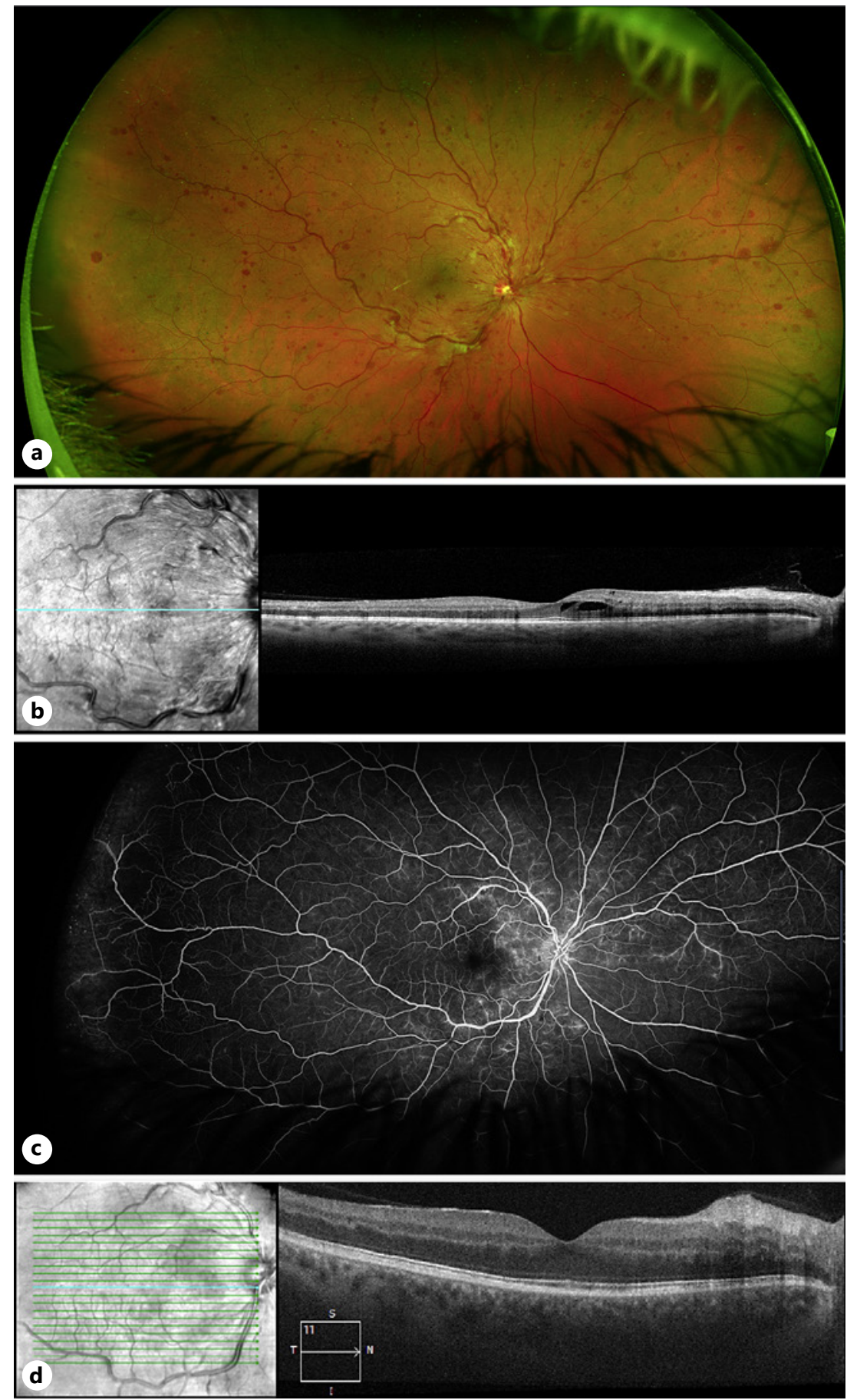

Fig. 1. a Wide-field color fundus photo shows typical findings of CRVO including mild optic disc edema, diffuse intraretinal hemorrhages, and cotton wool spots in the macula, retinal venous tortuosity, and intraretinal hemorrhages throughout the periphery. An OCT of the macula demonstrates cystoid macular edema partially involving the foveal center (b) and wide-field fluorescein angiogram demonstrates diffuse small vessel leakage posteriorly and capillary nonperfusion peripherally (c). d A follow-up OCT of the macula shows resolution of cystoid macular edema following treatment. CRVO, central retinal vein occlusion; OCT, optical coherence tomography. 


\section{Case Reports in Ophthalmology}

Table 2. Results of laboratory testing

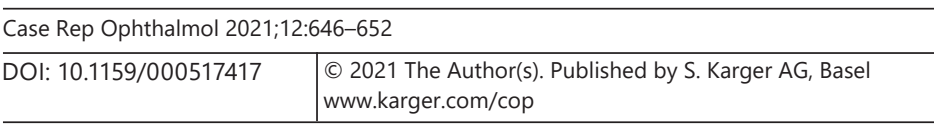

Miller and Kim: Central Retinal Vein Occlusion in a 46-Year-Old Man with COVID-19

\begin{tabular}{lc}
\hline Test & Result (reference range) \\
\hline Basic laboratory panel & \\
Complete blood count & All values within normal range \\
Complete metabolic panel & All values within normal range \\
Lipid panel & All values within normal range \\
Hypercoagulability panel & \\
PT & $11.4(9.4-12.5) \mathrm{s}$ \\
INR & $1(0.8-1.1)$ \\
PTT & $30.9(25.1-36.5) \mathrm{s}$ \\
Anticardiolipin IgG (GPL units) & $5.5(0.0-11.0)$ \\
Anticardiolipin IgM (MPL units) & $8.5(0.0-12.0)$ \\
Anti-ß2 glycoprotein 1 IgG & $0.5(0.0-7.9)$ \\
Anti-ß2 glycoprotein 1 IgM & $2.0(0.0-12.9)$ \\
Tissue thromboplastic inhibition test & $1.1(0.0-1.4) \mathrm{s}$ \\
dRVVT & $36.5(28.0-42.0) \mathrm{s}$ \\
Protein C functional & $92(83-168) \%$ \\
Protein S functional & $77(66-143) \%$ \\
SARS-CoV-2 antibodies, nucleocapsid & Reactive
\end{tabular}

IgG, immunoglobulin G; IgM, immunoglobulin M; MPL unit, cardiolipin-binding activity of $1 \mu \mathrm{g} / \mathrm{mLl}$ of affinity-purified IgM; GPL unit, cardiolipin-binding activity of $1 \mu \mathrm{g} / \mathrm{mL}$ of affinity-purified IgG; dRVVT, dilute Russell viper venom time; SARS-CoV-2, severe acute respiratory syndrome coronavirus 2 .

could be potentially specific to COVID-19 infection. He received an additional aflibercept injection, and his follow-up interval was extended.

\section{Discussion}

In the case reported here, a standard thrombophilia panel was unrevelatory. Although reported incidentally, our patient's COVID-19 antibody test returned positive, suggesting an etiology for his CRVO. This raised the possibility that our patient developed a CRVO during the acute phase of his infection with COVID-19, although he never developed respiratory symptoms or other signs associated with more severe disease. If such an association were observed on a population scale, there would be implications for our understanding of mildly symptomatic COVID-19 patients and thrombophilia.

Among 6 reported cases of RVO associated with COVID-19 identified in the literature, only 1 patient [7] was younger than 50 years and without additional risk factors (Table 1). Age, diabetes, and hypertension are the most common risk factors for RVO, and polycystic ovarian syndrome is recognized as a hypercoagulable state [18]. This is important to note, as despite the high prevalence of COVID-19 in the USA and across the world, an overall increased incidence of RVO has not been reported. There may however be a synergistic effect of preexisting RVO risk factors and infection with COVID-19. While the SARS-CoV-2 virus exhibits a propensity to infect type II pneumocytes, it has also been noted to infect perivascular cells in microvasculature outside the lung (kidney, small intestine, and myocardium) [19]. 
Detection of viral RNA in postmortem retinal biopsies leaves open the possibility that this occurs in the retinal circulation as well, but further study is needed to determine cell typespecific localization of the virus within the retina [20]. This represents a proposed mechanism by which SARS-CoV-2 could exhibit a direct effect on retinal endothelial cells and tip the scales toward retinal vein thrombosis in patients already at risk.

The importance of COVID-19 as a risk factor for systemic venous thrombosis has been well-established among patients hospitalized for hypoxemia or other systemic manifestations of COVID-19 infection. However, whether mildly symptomatic or even asymptomatic COVID-19 infection can lead to similar complications remains unclear. The patient presented here had constitutional symptoms nonspecific for COVID-19; however, he astutely sought out a COVID-19 serum antibody test, given the high prevalence of the virus during this time period. As this may have put him at increased risk for CRVO, this test result proved potentially relevant. Further study is warranted to address whether COVID-19 testing is appropriate for young patients diagnosed with RVO in the COVID-19 era.

\section{Statement of Ethics}

Written informed consent was obtained from the patient for publication of this case report and accompanying images. This single case report is exempt from ethics approval by policy of the University of Pennsylvania Institutional Review Board.

\section{Conflict of Interest Statement}

B.J.K. is a consultant for AsclepiX Therapeutics, Inc.

\section{Funding Sources}

The authors did not receive any funding related to this article.

\section{Author Contributions}

Both C.G.M. and B.J.K. contributed to the conception and design of this report as well as drafting and revision of the manuscript text.

\section{References}

1 Terpos E, Ntanasis-Stathopoulos I, Elalamy I, Kastritis E, Sergentanis TN, Politou M, et al. Hematological findings and complications of COVID-19. Am J Hematol. 2020;95(7):834-47.

2 Klok FA, Kruip MJHA, van der Meer NJM, Arbous MS, Gommers DAMPJ, Kant KM, et al. Incidence of thrombotic complications in critically ill ICU patients with COVID-19. Thromb Res. 2020;191:145-7.

3 Marinho PM, Marcos AAA, Romano AC, Nascimento H, Belfort R. Retinal findings in patients with COVID-19. Lancet. 2020;395(10237):1610.

4 Venkatesh P. Seeking clarity on retinal findings in patients with COVID-19. Lancet. 2020;396(10254): e36.

5 Vavvas DG, Sarraf D, Sadda SR, Eliott D, Ehlers JP, Waheed NK, et al. Concerns about the interpretation of OCT and fundus findings in COVID-19 patients in recent Lancet publication. Eye. 2020;34(12):2153-4.

6 Ouyang P, Zhang X, Peng Y, Jiang B. Seeking clarity on retinal findings in patients with COVID-19. Lancet. 2020; 396(10254):e35.

\section{Karger's}


7 Yahalomi T, Pikkel J, Arnon R, Pessach Y. Central retinal vein occlusion in a young healthy COVID-19 patient: a case report. Am J Ophthalmol Case Rep. 2020;20:100992.

8 Walinjkar JA, Makhija SC, Sharma HR, Morekar SR, Natarajan S. Central retinal vein occlusion with COVID-19 infection as the presumptive etiology. Indian J Ophthalmol. 2020;68(11):2572.

9 Gaba WH, Ahmed D, Al Nuaimi RK, Dhanhani AA, Eatamadi H. Bilateral central retinal vein occlusion in a 40-year-old man with severe coronavirus disease 2019 (COVID-19) pneumonia. Am J Case Rep. 2020;21: e927691.

10 Lorca DR, Fernandez AR, Santos MJ, Saenz-Frances F, Burgos-Blasco B, LopezJD. Bilateral retinal vein occlusion and diabetic retinopathy after COVID-19. Acta Ophthalmol. 2020.

11 Invernizzi A, Pellegrini M, Messenio D, Cereda M, Olivieri P, Brambilla AM, et al. Impending central retinal vein occlusion in a patient with coronavirus disease 2019 (COVID-19). Ocul Immunol Inflamm. 2020:1-3.

12 Sheth JU, Narayanan R, Goyal J, Goyal V. Retinal vein occlusion in COVID-19: a novel entity. Indian J Ophthalmol. 2020;68(10):2291.

13 Liu Q, Lahey JM, Karlen R, Stewart JM. Laboratory evaluation of hypercoagulable states in patients with central retinal vein occlusion who are less than 56 years of age. Retin. 2018;38(6):1175-9.

14 Fong ACO, Schatz H. Central retinal vein occlusion in young adults. Surv Ophthalmol. 1993;37(6):393-417.

15 Alexandra A0, Drenser KA. Hypercoagulable state in a patient with a retinal vein occlusion. Retin Cases Brief Rep. 2011;5(1):76.

16 Favresse J, Cadrobbi J, Eucher C, Elsen M, Laffineur K, Dogné J, et al. Clinical performance of three fully automated anti-SARS-CoV-2 immunoassays targeting the nucleocapsid or spike proteins. J Med Virol. 2020.

17 Roche. Elecsys ${ }^{\circledR}$ Anti-SARS-CoV-2. Package Insert 2020-07, V3.0; Material Numbers 09203095190 and 09203079190. US Food and Drug Administration. Undefined.

18 Okoroh EM, Hooper WC, Atrash HK, Yusuf HR, Boulet SL. Is polycystic ovary syndrome another risk factor for venous thromboembolism? United States, 2003-2008. Am J Obstet Gynecol. 2012;207(5):377.e1-8.

19 Ortega-Paz L, Capodanno D, Montalescot G, Angiolillo DJ. Coronavirus disease 2019-associated thrombosis and coagulopathy: review of the pathophysiological characteristics and implications for antithrombotic management. J Am Heart Assoc. 2021;10(3):e019650.

20 Casagrande M, Fitzek A, Püschel K, Aleshcheva G, Schultheiss H-P, Berneking L, et al. Detection of SARS-CoV-2 in human retinal biopsies of deceased COVID-19 patients. Ocul Immunol Inflamm. 2020;28(5):1-5. 